\title{
An in vivo model of echovirus-induced meningitis in neonates
}

\author{
Alexandra I. Wells ${ }^{1}$ and Carolyn B. Coyne ${ }^{1,2^{*}}$ \\ ${ }^{1}$ Department of Molecular Genetics and Microbiology, ${ }^{2}$ Duke Human Vaccine Institute, Duke \\ University School of Medicine, Durham, NC, USA
}

Running title: Model of echovirus meningitis

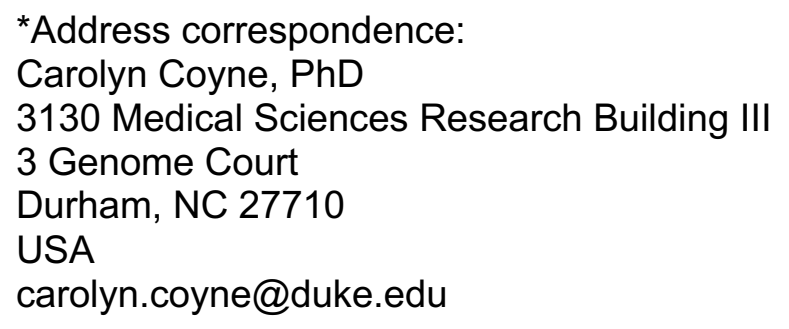




\section{Abstract}

Echoviruses are amongst the most common causes of aseptic meningitis worldwide, which can cause long-term sequelae and death, particularly in neonates. However, the mechanisms by which these viruses induce meningeal inflammation is poorly understood, owing at least in part to the lack of in vivo models that recapitulate this aspect of echovirus pathogenesis. Here, we developed an in vivo neonatal mouse model that recapitulates key aspects of echovirus-induced meningitis. We found that expression of the human homologue of the neonatal Fc receptor (FcRn), the primary echovirus receptor, in neonatal mice was not sufficient for infection of the brain. However, ablation of type I, but not III, IFN signaling in mice expressing human FcRn permitted high levels of echovirus replication in the brain, with corresponding clinical symptoms including delayed motor skills and hind limb weakness. We also defined the immunological response of the brain to echovirus infections and identified key cytokines induced by this infection. Lastly, we found that echoviruses robustly replicate in the leptomeninges, where they induce profound inflammation and cell death. Together, this work establishes an in vivo model of aseptic meningitis associated with echovirus infections and defines the specificity of echoviral infections within the meninges.

\section{Significance Statement}

Echoviruses are amongst the most common causes of aseptic meningitis worldwide, which can cause long-term sequelae or even death. The mechanisms by which echoviruses infect the brain are poorly understood, largely owing to the lack of robust in vivo models that recapitulate this aspect of echovirus pathogenesis. Here, we establish a neonatal mouse model of echovirusinduced aseptic meningitis and show that expression of the human homologue of the neonatal Fc receptor (FcRn), the primary receptor for echoviruses, and ablation of type I interferon (IFN) signaling are required to recapitulate echovirus-induced meningitis and clinical disease. These 
findings provide key insights into the host factors that control echovirus-induced meningitis and a model that could be used to test anti-echovirus therapeutics.

\section{Introduction}

Enteroviruses are the main causes of aseptic meningitis worldwide, which is characterized by meningeal inflammation not associated with any identifiable bacterial species in cerebrospinal fluid (CSF). Approximately $90 \%$ of aseptic meningitis cases in infants (1) and $50 \%$ in older children and adults (2) are caused by enteroviruses, with the group B enterovirus members coxsackievirus B (CVB) and echoviruses being amongst the most common $(3,4)$. Infants and young children are particularly vulnerable to complications of echovirus-associated neuronal complications, which can cause long-term sequelae including seizure disorders $(5,6)$, and are associated with high rates of mortality, which occurs in as many as one-third of cases $(7,8)$. Despite the clear association between echoviruses and aseptic meningitis, the mechanisms by which these viruses induce meningeal inflammation is poorly understood, owing at least in part to the lack of in vivo models that recapitulate this aspect of echovirus pathogenesis.

Echoviruses are the largest subgroup of the Enterovirus genus and consist of approximately 30 serotypes. We and others have shown that the neonatal Fc receptor $(\mathrm{FcRn})$ is the primary receptor for echoviruses $(9,10)$. FcRn transports and regulates the circulating halflife of immunoglobulin $G(\lg G)$ and albumin and is enriched in the endothelium of the central nervous system (CNS), including the blood-brain barrier (BBB), where it mediates the efflux of IgG from the brain (11-15). Although several studies have investigated the possible mechanistic basis for CVB-associated neuronal dysfunction in vitro and in vivo (16-18), much less is known about echovirus-associated CNS complications. Intracerebral inoculation of newborn mice expressing VLA-2, a reported receptor for echovirus 1, exhibit paralysis and motor defects (19). Other work suggests that type I interferons (IFNs) play a role in echovirus $11 \mathrm{CNS}$ disease following intracranial inoculation (20). We previously generated an in vivo mouse model of 
echovirus 11 pathogenesis using adult and neonatal mice and showed that expression of the human homologue of FcRn in mice lacking type I IFN signaling were susceptible to echovirus infection, including infection of the brain (21). However, the consequences of echovirus infection including the induction of cytokines and cell death in the brains of these mice, as well as identification of the region(s) of the brain targeted by infection were not explored.

The meninges surround the brain and are composed of three distinct membranous layers which includes the dura, arachnoid, and pia mater. A hallmark of aseptic meningitis involves inflammation of the meninges, resulting in immune cell infiltration and swelling. In human cases of confirmed echovirus aseptic meningitis, infection is associated with a robust inflammatory response, as indicated by the presence of high levels of proinflammatory mediators in CSF (2224). Higher levels of type I IFNs are also present in CSF isolated from enterovirus-associated meningitis than from bacterial meningitis (25), suggesting that these IFNs play a prominent role in aseptic meningitis. Type I IFNs, which include IFN $\alpha$ s and IFN- $\beta$, provide key antiviral defenses from many neurotropic viruses, including flaviviruses, alphaviruses, and herpesviruses (26). In some cases, type III IFNs (IFN- $\lambda$ s) also defend from CNS viral infections $(27,28)$ and have been proposed to function by alterations in BBB permeability (27). Whether type I and III IFNs play differential roles in echovirus infections of the CNS is unknown.

Here, we developed an in vivo model of echovirus-induced aseptic meningitis in neonatal mice which recapitulates many of the disease manifestations observed in humans. We show that expression of human FcRn alone is not sufficient to mediate echovirus infection of the brains of 7-day-old neonatal mice. In addition, the brains of mice deficient in either type I or III IFN signaling alone were not permissive to echovirus infection. In contrast, we found that humanized FcRn mice deficient in IFNAR, the type I IFN receptor, but not IFNLR, the type III IFN receptor, exhibited high levels of echovirus replication in the brain, with corresponding clinical symptoms including hind limb weakness and paralysis. Using this model, we defined the immune response in the brains of 
echovirus-infected mice, which included the induction of high levels of IL-6, CXCL10, and granulocyte colony-stimulating factor (GSF)-3. Lastly, we show that echoviruses replicate in the leptomeninges and induce inflammation and cytotoxicity in these membranes, including activation of apoptotic cell death. Together, this work establishes an in vivo model of aseptic meningitis associated with echovirus infections and defines the specificity of echoviral infections in the meninges.

\section{Results}

Ablation of type I interferon signaling and human FcRn expression are required for echovirus infection of the brain

Previously, we developed a mouse model of echovirus pathogenesis through intraperitoneal (IP) inoculation using mice expressing the human homologue of $\mathrm{hFcRn}\left(\mathrm{hFcRn}{ }^{\mathrm{Tg} 32}\right)$ that are also ablated in type I IFN signaling by deletion of IFNAR (hFcRn ${ }^{\top 932}$ IFNAR $\left.^{-/-}\right)(21)$. hFcRn ${ }^{\top 932}$ mice are deficient in expression of mouse FcRn and express human FcRn under the control of the native human promotor (29). To determine if type III IFNs also play a role in echovirus infections at secondary sites of infection including the brain, we generated $\mathrm{hFcRn} \mathrm{Tg}^{\mathrm{Tg} 32}$ mice deficient in IFNLR expression, the receptor for type III IFNs (hFcRn ${ }^{T g 32}$-IFNLR ${ }^{-/}$) (30). We used six genotypes of mice, including the $\mathrm{hFcRn}$ mice described above $\left(\mathrm{hFcRn}{ }^{T g 32}, \mathrm{hFcRn}^{\mathrm{Tg} 32}{ }_{-\mathrm{IFNAR}}{ }^{-/-}\right.$, and $\mathrm{hFcRn}^{\mathrm{Tg} 32}$. $\mathrm{IFNLR}^{-/-}$) and animals expressing murine FcRn that were immunocompetent (C57/BL6, WT) or deficient in type I or III IFN signaling (IFNAR ${ }^{-/-}$or IFNLR ${ }^{-/-}$, respectively) (Figure 1A). Neonatal (7day old) mice were inoculated with $10^{4}$ PFU of echovirus 5 (E5) by the IP route and monitored for three days post-inoculation (dpi). We observed death in $100 \%$ of $\mathrm{hFCRn}^{\text {Tg32-IFNAR }}{ }^{-{ }^{--}}$animals by 2 dpi (Figure 1B). In contrast, there were no clinical symptoms of illness in any other genotype and all animals survived until 3dpi (Figure 1B). There were no significant differences in mortality between male and female $\mathrm{hFCRn}^{\mathrm{Tg} 32}$-IFNAR ${ }^{-/-}$mice (Supplemental Figure 1A). We next determined the level of circulating virus in the blood and brains of these animals. We detected 
high levels of E5 in the blood (6 of 6 mice) and brains (12 of 12 mice) of hFcRn ${ }^{\text {Tg32 }}{ }_{\text {IFNAR }}^{-/-}$mice, but no detectable virus in any other genotype (Figure 1C, 1D). There were no significant differences in E5 titer in the brains of male or female $\mathrm{hFcRn}{ }^{T g 32}$-IFNAR ${ }^{-/-}$mice (Supplemental Figure 1B).

Consistent with our previous findings with E11 (21), we found that there was robust replication in the livers and pancreases of E5 infected hFcRn ${ }^{T g 32}{ }_{-I F N A R}{ }^{-1-}$ mice (12 of 12 mice) (Supplemental Figure 1C, 1D). However, in contrast to the brain, we found that $\mathrm{hFcRn}{ }^{\mathrm{Tg} 32}$ $\mathrm{IFNLR}^{-/-}$animals also contained virus in the liver (4 of 8 mice) and pancreas (8 of 8 mice) (Supplemental Figure 1C, 1D). We also detected low to mid-levels of E5 in the pancreases of immunocompetent $\mathrm{hFcRn}{ }^{\mathrm{Tg} 32}$ mice ( 5 of 5 mice) and to a much lesser extent in liver ( 1 of 5 mice) (Supplemental Figure 1C, 1D). There was no detectable virus in any animals expressing the murine homologue of FcRn (Supplemental Figure 1C, 1D), consistent with our previous work (21). Collectively, these data show that echovirus infections in the brain require expression of $\mathrm{hFCRn}$ and that the primary barrier to infection is type I IFN-mediated signaling.

\section{Echovirus infections cause paralysis and motor defects in infected mice}

Because we observed high levels of mortality in mice infected with $10^{4}$ PFU E5 by the IP route, we investigated the neurotropism and neurovirulence of E5 in mice infected with a lower inoculum ( $10^{3}$ PFU) of E5 by the IP route and monitored animals daily for 2 days. At this lower inoculum we observed death in approximately $50 \%$ of $\mathrm{hFcRn}^{\mathrm{Tg} 32}$-IFNAR ${ }^{-/-}$animals by $1.5-2$ days dpi compared to no mortality in $\mathrm{hFcRn}{ }^{\mathrm{Tg} 32}$-IFNLR ${ }^{-/-}$animals (Supplemental Figure 2A). There were no differences in mortality between male and female $\mathrm{hFcRn}^{\mathrm{Tg} 32}$-IFNAR ${ }^{-/-}$mice (Supplemental Figure 2C). Infected animals were monitored for signs of illness (e.g., delayed movements, paralysis, discoloration, lack of nursing, lack of parental care, and death) throughout the duration of infection. There were no signs of clinical illness in echovirus-infected $\mathrm{hFcRn}{ }^{\top \mathrm{Tg} 32}{ }_{\text {IFNLR }}{ }^{-/}$animals at 1 or 2 dpi (Figure 1E). There were also no obvious clinical symptoms in hFcRn Tg32-IFNAR ${ }^{-/-}$ 
animals at $1 \mathrm{dpi}$. However, by $1.5-2 \mathrm{dpi}$, there were clear defects in motor skills and various degrees of paralysis in $\mathrm{hFcRn}{ }^{\top \mathrm{g} 32}-\mathrm{IFNAR}^{-/-}$infected animals. There were a range of defects ranging from mild loss of motor function in one or more limb characterized by difficulty walking, hemiplegia that obstructed mobility or hind limb paralysis (Figure 1E and Supplemental Figure 2B). To correlate clinical symptoms with infection, we titrated virus from the brains of infected animals at 1 and $2 \mathrm{dpi}$, which revealed similar titers at both days in brain and higher titers in blood at $2 \mathrm{dpi}$ in $\mathrm{hFcRn}^{\mathrm{Tg} 32}$-IFNAR ${ }^{-/}$animals (Figure 1F). There were no differences in titers between male and female mice (Supplemental Figure 2D). Additionally, similar to the lack of clinical disease and low morality rates, no virus was detected in the blood or brains of hFcRn ${ }^{T g 32}{ }^{-I F N L R}{ }^{-}$ I- animals (Figure 1F). These data show that echoviruses induce clinical symptoms of neurological disease, which occurs in an hFcRn-dependent manner and requires ablation of type I IFN signaling.

\section{Immunological signature of echovirus infected brains}

To define the immunological signature of echovirus infected brains, we harvest the brains of E5infected mice expressing hFcRn (hFcRn ${ }^{T g 32}, \mathrm{hFcRn}{ }^{\text {Tg32 }}-\mathrm{IFNAR}^{-/-}$, and $\left.\mathrm{hFcRn}{ }^{\mathrm{Tg} 32}-\mathrm{IFNLR}^{-/-}\right)$at $3 \mathrm{dpi}$ and performed multianalyte Luminex-based profiling of 27 cytokines and chemokines on brain tissue homogenates. We found that echovirus infection induced high levels of cytokines in brain tissue of $\mathrm{hFCRn}^{\mathrm{Tg} 32}$-IFNAR ${ }^{-/-}$infected mice at 3dpi, including high levels of Granulocyte colonystimulating factor-3 (GSF-3), which was the most abundant cytokine detected ( 750-fold over uninfected) (Figure 2A, 2C). Other highly induced cytokines included IL-6 (25-fold over uninfected), CXCL10 (17-fold over uninfected), monocyte chemoattractant protein-1 (MCP-1, 16fold over uninfected), and keratinocyte-derived chemokine (KC, 12-fold over uninfected) (Figure 2A, 2D, 2E, 2F). There was no significant induction of any cytokines in infected $\mathrm{hFcRn} \mathrm{Tg}^{\mathrm{Tg} 32}$ or $\mathrm{hFcRn}^{\text {Tg32-IFNLR }}{ }^{-/}$animals at 3dpi (Figure 2A). We did not detect the type I IFNs IFN- $\alpha 2$ or IFN$\beta$ or the type III IFN IFN- $\lambda 1$ in the brains of any mice (Supplemental Figure 3A-C). 
We next assessed the kinetics of cytokine induction in echovirus infected brains isolated from $\mathrm{hFCRn}^{\text {Tg32 }}$ IFNAR $^{-/-}$and $\mathrm{hFCRn}{ }^{\text {Tg32 }}$ IFNLR $^{-/-}$infected animals at 1 and 2 dpi. Consistent with the overall low levels of virus in the brains of mice at 1dpi, we observed very little cytokine induction in $\mathrm{hFcRn}^{\mathrm{Tg} 32}$-IFNAR ${ }^{-/}$animals at this time (Figure 2B). However, by 2dpi, there were high levels of similar cytokines observed at 3dpi, including G-CSF (647-fold over uninfected), CXCL10 (33-fold over uninfected), IL-6 (28-fold over uninfected), and MCP-1 (23-fold over uninfected) (Figure 2B, 2G-J). This cytokine induction paralleled the accumulation of viral RNA (vRNA) in the brain, as assessed by RT-qPCR for vRNA and the transcript for CXCL10 (Supplemental Figure 3D-E). There were no cytokines significantly induced in infected $\mathrm{hFCRn}^{\mathrm{Tg} 32}$-IFNLR ${ }^{-/-}$animals at either time point (Figure 2B). These data show that echovirus infection of brain tissue results in an immunological response characterized by the induction of select pro-inflammatory cytokines and chemokines.

\section{Echoviruses replicate in the leptomeninges to induce meningeal inflammation}

Very little is known regarding the cellular or structural targets of echovirus infections in the brain, including the meninges, which is composed of three membranous layers. To define the site(s) of echovirus replication in the brain, we infected $h F c R n^{T g 32}, h_{F c R n}{ }^{T g 32}-I F N A R^{-/-}$, and $h F c R n^{T g 32}$ IFNLR $^{-/-}$mice with $10^{4}$ PFU E5 for $3 d$ to maximize infection. At this time, whole brains were removed, sectioned, and processed for hybridization chain reaction (HCR) using E5-specific probes. HCR allows for fluorescent quantitative RNA detection with enhanced sensitivity over conventional hybridization approaches given signal amplification resulting from the self-assembly of secondary detection hairpins into amplification polymers $(31,32)$. Whole brain confocal microscopy-based tile scanning of $\sim 36 \mathrm{~mm}^{2}$ was then performed to define the region(s) and cell(s) infected by E5. We observed high levels of E5 vRNA in infected hFcRn ${ }^{\text {Tg32 }}{ }_{-I F N A R}{ }^{-/-}$brain tissue, but none in $\mathrm{hFcRn}^{\mathrm{Tg} 32}$ or $\mathrm{hFcRn}{ }^{\mathrm{Tg} 32}$-IFNLR ${ }^{-/}$brains, at 3dpi, which was localized to a distinct region surrounding the brain (Figure 3A, Supplemental Figure 4A, 4B). This region was specific for the 
leptomeninges, which includes the two inner layers of the meninges (Figure 3B, 3C). In addition, there were concentrated regions of high levels of E5 vRNA surrounding blood vessels localized throughout the meninges (Figure 3D). Although the choroid plexus has been reported to express high levels of FcRn (15), we did not detect any infection in this region (Supplemental Figure 4C). To determine the timing of E5 vRNA in the meninges, we performed HCR in whole brains isolated from $\mathrm{hFCRn}{ }^{\text {Tg32 }}$ IFNAR $^{-1-}$ mice infected with E5 for 1-3dpi. At 1dpi, we found that the levels of E5 VRNA were undetectable like uninfected controls, but by $2 \mathrm{dpi}$, there were clear areas of E5 infection, which significantly increased by 3dpi (Figure 3E, 3F).

To define the localization of FcRn within the brains of $\mathrm{hFcRn}{ }^{T g} 32$ mice and to correlate this expression with E5 infection, we performed immunohistochemistry for $\mathrm{hFcRn}$ in $\mathrm{hFcRn}{ }^{\mathrm{Tg} 32}{ }^{-I F N A R^{-}}$ I- mice. We found that $\mathrm{hFcRn}$ was enriched in the leptomeninges of infected animals, where it was concentrated to regions associated with high levels of vRNA (Figure 4A).

Next, we assessed the impacts of E5 infection on the integrity of the meninges. We noted instances of acute meningitis and inflammatory tissue damage in $\mathrm{hFcRn}{ }^{\mathrm{Tg} 32}$-IFNAR ${ }^{-/-}$mice infected with E5 for 3dpi, which included areas of immune cell infiltration (Figure 4B). Areas of inflammation correlated with high levels of E5 replication, as indicated by aligning HCR and H\&E images (Figure 4C). Lastly, to determine whether E5 infection induces direct cell death or damage to the meninges, we performed immunohistochemistry for cleaved caspase-3, which revealed discrete areas of apoptosis as early as 2dpi, with more significant levels at 3dpi (Figure 4D). Together, these data show that echovirus infection of the meninges induces pronounced tissue damage and inflammation.

\section{Discussion}

Echovirus infections are common causes of neonatal meningitis, which can be fatal. However, how these viruses infect the brain and their regional tropism within the brain remains unknown. Here, we developed an in vivo model of echovirus-induced meningitis and used this 
model to define the cellular targets and host immune pathways associated with echovirus infection within the brain. We show that expression of the human homologue of FcRn is necessary, but not sufficient, for echovirus infection of the brain. In addition, we show that type I IFNs, but not type III IFNs, provide a barrier to echovirus infections of the meninges and that ablation of this pathway sensitizes the brain to infection, which induces a robust immune response. Lastly, we define the specificity of echovirus replication in the brain and show that high levels of replication in the leptomeninges induced inflammation and cell death. These studies thus provide key insights into the events associated with echovirus-induced meningitis and an in vivo model that could be used to test echovirus therapeutics targeting echovirus-induced neuronal disease.

FcRn is expressed on the microvasculature of the blood-brain barrier (15), where it has been proposed to mediate the efflux of antibodies out of the brain (33). In addition to this localization, FcRn is expressed in the epithelium of the choroid plexus (15). This pattern of expression is conserved between humans and in $\mathrm{hFcRn}{ }^{\top \mathrm{g} 32}$ mice (34). In addition to these sites, our data show that FcRn is also expressed at distinct regions of the meninges. The meninges are composed of diverse cell types, with distinct patterns of expression regionally between the dura, arachnoid layer, and pia mater, with the arachnoid and pia mater referred to as the leptomeninges (35). The meningeal layers are primarily comprised of fibroblasts, with each subtype expressing their own unique transcriptional signature (35). In addition to fibroblasts, the arachnoid layer also contains arachnoid barrier cells, which are epithelial-like in origin and separate the dura mater from the subarachnoid space in which CSF accesses the brain. The meninges also contain a large population of immune cells, including $\mathrm{CD}^{+} \mathrm{T}$ cells and $\mathrm{B}$ cells. Notably, meningeal macrophages are a specialized subclass of macrophages which are long-lived in the leptomeninges (36). We observed the highest levels of E5 infection restricted to the leptomeninges, the inner layer of the meninges containing the pia mater and arachnoid layers, which also expressed high levels of FcRn. Given the robust levels of E5 infection in the meninges, it is likely that fibroblasts are at least one target of infection. However, it is possible that other cell 
types, such as macrophages, are also permissive to infection and contribute to pathogenesis. Collectively, these data suggest that the regional expression of FcRn plays a direct role in the tropism of echoviruses in the brain and directly correlates with their high levels of replication in the meninges.

Several studies have investigated the levels of cytokines and chemokines within the CSF of infants and children with confirmed echovirus-induced meningitis. The CSF of children with echovirus 30-induced meningitis contain very high levels of MCP-1 and IL-6 compared to controls $(23,37)$. Our data show that $h F c n^{T g 32}$-IFNAR ${ }^{-/-}$animals also induce very high levels of MCP-1 and IL-6 in brain tissue homogenates. MCP-1, also known as CCL2, is a monocyte chemoattractant responsible for recruiting monocytes and dendritic cells to the site of inflammation due to infection. IL-6 is a proinflammatory cytokine that is secreted by macrophages that have detected a viral infection. These immune mediators play important roles in alerting the immune system to a viral infection and recruiting immune cells to the sites of infection. However, because we used brain tissue homogenates and human studies can are restricted to CSF, it is unclear which cytokines induced by echovirus infections mediate the influx of immune cells observed in echovirus-induced meningitis. Although the differential roles of type I and III IFNs has been established in the respiratory and intestinal epithelium during enterovirus infection $(30,38-$ 40), whether these IFNs play distinct roles in enterovirus-induced meningitis has remained unclear. Our data suggest that type I IFNs are the sole barrier to echovirus infection of the CNS, which is consistent with previous work defining indicating that these IFNs form at least one bottleneck to poliovirus access to the CNS (41). However, type I IFNs were not detected in multiplex Luminex profiling of brain homogenates from echovirus-infected mice, suggesting that these IFNs are not produced locally in the brain. It is therefore likely that circulating IFNs produced at distal sites, such as the liver, trigger antiviral responses in the CNS that protect from echovirus infections. 
In humans, neonates are at increased risks for echovirus-induced morbidity and mortality. For example, rates of echovirus-induced paralysis decreased with increased age at inoculation (42). Our previous work showed that adult echovirus-infected mice exhibit low levels of infection in the brain following IP inoculation (21). Our data presented here show that neonatal mice are highly sensitive to echovirus-induced neuronal dysfunction and contain high levels of viral infection within the meninges. The mechanistic basis for age-related differences in echovirus infection of the brain remains unclear. Given that BBB forms in mice prior to birth (43), this barrier is unlikely to participate in these age-related differences. Age-related differences in neuronal susceptibility to infections not specific to echoviruses and has also been shown for reovirus (44). However, unlike this work, our data suggest that type I IFNs are not the primary drivers for differential age susceptibility given that both adult and neonatal animals in our work were deficient in IFNAR expression. Other work has shown that age-related differences in susceptibility to cardiac infections by CVB may result from differences in receptor expression $(45,46)$. Age-related differences in FcRn expression in humans are unknown, but its expression in human liver is not dependent on age (47), suggesting this is unlikely to explain age-related differences in echovirusassociated neuronal infections. Therefore, it remains unclear which factors contribute to agerelated difference in infection of the CNS.

Our findings presented here define key aspects of echovirus infection of the brain, including the sites of viral replication and the consequences of this infection. We show that FcRn is necessary but not sufficient for echovirus infection of the brain in vivo and that type I IFNs control infection within the meninges. Collectively, these studies develop a model of echovirus aseptic meningitis, which could aid in the testing of novel therapeutics.

\section{Materials and Methods}

Cell lines and viruses. HeLa cells (clone 7B) were provided by Jeffrey Bergelson, Children's 335 Hospital of Philadelphia, Philadelphia, PA, and cultured in MEM supplemented with 5\% FBS, non- 
essential amino acids, and penicillin/streptomycin. Experiments were performed with echovirus 5 (Noyce strain, E5), which was obtained from the ATCC. Virus was propagated in HeLa cells and purified by ultracentrifugation over a $30 \%$ sucrose cushion, as described previously (48). All viruses were sequenced for viral stock purity following propagation. Purity of all viral stocks was confirmed by Sanger sequencing of VP1 using enterovirus-specific primers (49). Briefly, RNA extraction was performed on $10 \mu$ of purified virus stock, according to manufacturer's instructions (Qiagen Cat. 529904). RNA was reverse transcribed using SuperScript III reverse transcription kit, (Invitrogen cat. 18080093) according to manufacturer's instructions, with a pan enterovirus primer (vir21; ATAAGAATGCGGCCGCTTTTTTTTTTTTTTTTTTTTTTTTT), followed by an RNaseH treatment for 20 minutes at $37^{\circ} \mathrm{C}$. PCR was performed with $5 \mu$ l of the cDNA reaction using BioRad iTaq DNA polymerase (BioRad cat. 1708870). Virus specific primers are as follows: E5 forward 5'TATCGCCAATTACAACGCGAA-3'; E5 reverse 5'TTGGTTTGAAGTAAACCCTTA-3'.

Animals. All animal experiments were approved by the Duke University Animal Care and Use Committees, and all methods were performed in accordance with the relevant guidelines and

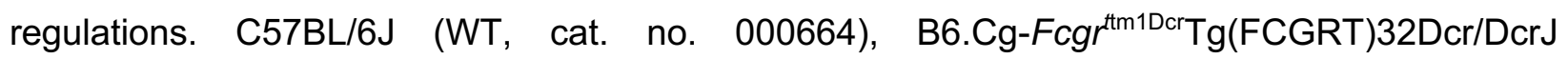
(hFcRn ${ }^{T g 32}$, cat. no. 014565), and B6.(Cg)-Ifnar1 ${ }^{\text {tm1.2Ees } / J ~(I F N A R ~}{ }^{-/}$, cat. no. 028288) mice were purchased from The Jackson Laboratory. $\mathrm{hFCRn}^{\mathrm{Tg} 32}$-IFNAR ${ }^{-/-}$mice were generated as described previously (21). B6.Ifnlr $\mathrm{r}^{-/} / \mathrm{J}\left(\mathrm{IFNLR}^{-/-}\right)$mice were provided by Dr. Megan Baldridge (Washington University School of Medicine). hFcRn ${ }^{\top g 32}-I F N L R^{-/-}$mice were generated by crossing B6.Cg-

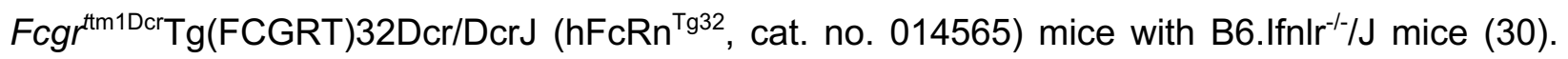
Breeders were established that were deficient in mouse FcRn and IFNLR and were homozygous for the hFcRn transgene. All animals used in this study were genotyped by Transnetyx. 
Suckling pup infections. 7 -day-old mice were inoculated by the IP route with $10^{4}$ or $10^{3} \mathrm{PFU}$ of E5. Inoculation was performed using a $1 \mathrm{~mL}$ disposable syringe and a 27 -gauge needle in $50 \mu \mathrm{L}$ of 1X PBS. Mice were euthanized at either 1-, 2-, or 3-days post inoculation and organs harvested into $0.5 \mathrm{~mL}$ of DMEM and stored at $-80^{\circ} \mathrm{C}$. Tissue samples for viral titration were thawed and homogenized with a TissueLyser LT (Qiagen) for 5 minutes, followed by brief centrifugation for 5 minutes at $8000 x g$. Viral titers in organ homogenates were determined by TCID50s in HeLa cells and enumerated following crystal violet staining.

Luminex assays. Luminex profiling was performed on whole brain tissue homogenates where were homogenized with a TissueLyser LT (Qiagen) for 5 minutes, followed by a centrifugation of $10,000 x g$ for 10 minutes. Luminex kits that were used are as follows a custom mouse IFN kit (IFN alpha, IFN beta, IL-28, Invitrogen), mouse cytokine 23-plex (Bio-Rad, M60009RDPD), and mouse CXCL10 (Invitrogen, EPX01A-26018-901), according to the manufacturer's protocol. Assays were read on a BioPlex 200 by BioRad. Heat maps were generated using the fold change in concentration (picograms/milliliter) of each animal compared to the average of uninfected animals and was made in GraphPad Prism. Violin plots are shown as the concentration for each animal (one point) in picograms/milliliter.

RNA Extraction and RT-qPCR. Total RNA was isolated from brains using the Sigma GenElute Total Mammalian RNA Miniprep Kit (Sigma, RTN350), according to the manufacturer protocol with the addition of a Sigma DNase digest reagent (Sigma, DNASE70). RNA (1 ug total) was reverse transcribed using iScript cDNA Synthesis Kit (Bio-Rad, 1708891) and diluted to $100 \mathrm{ul}$ in $\mathrm{ddH}_{2} \mathrm{O}$ for subsequent qPCR analyses. RT-qPCR was performed using the iTaq Universal SYBR Green Supermix (Bio-Rad, 1725121) on a CFX96 Touch Real-Time PCR Detection System (BioRad, 1855195). Primer sequences can be found in Supplemental Table 1. 
Immunohistochemistry. Tissues were fixed in $10 \%$ buffered formalin for $24 \mathrm{hrs}$ and then transferred to $70 \%$ ethanol. Tissues were embedded in paraffin and sectioned. Slides were stained with cleaved caspase 3 (Asp175) (9661, Cell Signaling) or human FCGRT (Abcam, ab139152). Tissue sections were deparaffinized with xylene and rehydrated with decreasing concentrations of ethanol $(100 \%, 95 \%, 80 \%)$, then washed with $\mathrm{ddH}_{2} \mathrm{O}$. Antigen unmasking was performed with slides submerged in $10 \mathrm{mM}$ citrate buffer $(\mathrm{pH} 6.0)$ and heated in a steamer for 20 minutes at $\sim 90^{\circ} \mathrm{C}$. Slides were cooled to room temperature and slides were immunostained with cleaved caspase 3 or FCGRT using Vectastain Elite ABC HRP (Vector Biolabs, PK-6100), according to the manufacturer's instructions. Slides were incubated in $6 \% \mathrm{H}_{2} \mathrm{O}_{2}$ in methanol for 30 min then washed 3 times for 5 minutes in $\mathrm{H}_{2} \mathrm{O}$. Avidin block (Vector, SP-2001) was applied for 15 minutes and washed twice in $\mathrm{H}_{2} \mathrm{O}$ followed by biotin block (Abcam, ab156024) for 15 minutes and washed twice in $\mathrm{H}_{2} \mathrm{O}$. Finally, serum-free protein block was applied for 10 minutes, and cleaved caspase 3 antibody was diluted 1:100 and the FCGRT antibody was diluted to 1:200 in TBS-T (Tris-buffered saline, $0.1 \%$ Tween 20) and slides incubated overnight in a humidified chamber at 4C. Next, slides were washed three times for 5 min in PBST and exposed to the goat anti-rabbit biotinylated secondary antibody (Vector, BA-1000) for $30 \mathrm{~min}$. Slides were rinsed in PBST three times for $5 \mathrm{~min}$ and the Vectastain Elite ABC HRP kit was applied for $30 \mathrm{~min}$. Slides were rinsed in PBST for three times for $5 \mathrm{~min}$ and diaminobenzidine substrate for 5 mins, which was terminated with water incubation. Slides were counterstained with hematoxylin for $1 \mathrm{~min}$, thoroughly rinsed with $\mathrm{H}_{2} \mathrm{O}$, and incubated in $0.1 \%$ sodium bicarbonate in $\mathrm{H}_{2} \mathrm{O}$ for 5 mins. Slides were then dehydrated with increasing concentrations of ethanol, cleared with xylene and mounted with Cytoseal 60 (Thermo Scientific, 83104). Images were captured on an IX83 inverted microscope (Olympus) using a UC90 color CCD camera (Olympus). 
HCR and Imaging. HCR was performed following the Molecular Instruments HCR v3.0 protocol for FFPE human tissue sections $(31,32)$. Briefly, tissue sections were deparaffinized with xylene and rehydrated with decreasing concentrations of ethanol (100\%, 95\%, 80\%). Antigen unmasking was performed with slides submerged in $10 \mathrm{mM}$ citrate buffer $(\mathrm{pH} \mathrm{6.0)}$ and heated in a steamer for 20 minutes at $\sim 90^{\circ} \mathrm{C}$. Slides were cooled to room temperature. Sections were treated with 10 $\mu \mathrm{g} / \mathrm{mL}$ Proteinase $\mathrm{K}$ for $10 \mathrm{~min}$ at $37^{\circ} \mathrm{C}$ and washed with RNase free water. Samples were incubated for 10 minutes at $37^{\circ} \mathrm{C}$ in hybridization buffer. Sections were incubated overnight in a humidified chamber at $37^{\circ} \mathrm{C}$ with $3 \mathrm{pmol}$ of initiator probes in hybridization buffer. We designed probes for E5 (Supplemental Table 2). The next day, slides were washed in probe wash buffer and 5x SSCT for 4x 15 min, according to the manufacturer's instructions. Samples were incubated in a humidified chamber at $37^{\circ} \mathrm{C}$ for 30 minutes in amplification buffer. Fluorescent hair pins were heated to $95^{\circ} \mathrm{C}$ for 90 seconds and snap cooled at room temperature for $30 \mathrm{~min}$. Hairpins and amplification buffer were added to the sample and incubated overnight at room temperature. Hairpins were washed off with $5 x$ SSCT for 5 minutes, 15 minutes, 15 minutes, and 5 minutes followed by a wash with PBS containing DAPI. Slides were mounted in Vectashield with DAPI. Slides were imaged on a Zeiss 880 with Airyscan inverted confocal microscope. Image analysis was performed using FIJI.

Statistics. All statistical analysis was performed using GraphPad Prism version 9. Data are presented as mean \pm SD. Parametric tests were applied when data were distributed normally based on D'Agostino-Pearson analyses; otherwise, nonparametric tests were applied. The logrank test was used to analyze the statistical difference of the survival rate in kaplan meier curves. In most cases, a Kruskal-Wallis test with a Dunn's test for multiple comparisons or Two-way Anova with Šídák's multiple comparisons tests were used to determine statistical significance, as described in the figure legends. $\mathrm{P}$ values of $<0.05$ were considered statistically significant, ${ }^{*} p<0.05,{ }^{* *} p<0.005,{ }^{* * *} p<0.0005,{ }^{* * *} p<0.0001$, as noted in figure legends. 
439 We thank Cristian Ovies and Kalena Grimes (Duke University School of Medicine) for technical 440 assistance, Megan Baldridge (Washington University) for providing IFNLR ${ }^{-/-}$mice, and Sujan 441 Shresta (La Jolla Institute for Immunology) for providing hFcRn ${ }^{T g 32}$ IFNAR $^{-/-}$mice. This project 442 was supported by NIH R01-Al150151 (C.B.C), NIH T32-AI060525 (A.I.W), NIH F31-Al149866 443 (A.I.W). The funders had no role in study design, data collection and analysis, decision to publish, 444 or preparation of the manuscript.

445

446

447

448

449

450

451

452

453

454

455

456

457

458

459

460

461

462

463

464

465

466

467

468

469

470

471

472

473

474

475

476 


\section{Literature Cited}

1. L. E. Berlin et al., Aseptic meningitis in infants < 2 years of age: diagnosis and etiology. $J$ Infect Dis 168, 888-892 (1993).

2. C. Huang et al., Multiple-year experience in the diagnosis of viral central nervous system infections with a panel of polymerase chain reaction assays for detection of 11 viruses. Clin Infect Dis 39, 630-635 (2004).

3. H. A. Rotbart, Enteroviral infections of the central nervous system. Clin Infect Dis 20, 971-981 (1995).

4. C. Centers for Disease, Prevention, Outbreaks of aseptic meningitis associated with echoviruses 9 and 30 and preliminary surveillance reports on enterovirus activity--United States, 2003. MMWR Morb Mortal Wkly Rep 52, 761-764 (2003).

5. H. Y. Lee et al., Clinical features of echovirus 6 and 9 infections in children. J Clin Virol 49, 175-179 (2010).

6. H. J. Kim et al., Epidemics of viral meningitis caused by echovirus 6 and 30 in Korea in 2008. Virol J 9, 38 (2012).

7. Y. Y. Chuang, Y. C. Huang, Enteroviral infection in neonates. J Microbiol Immunol Infect 52, 851-857 (2019).

8. J. F. Modlin, Perinatal echovirus infection: insights from a literature review of 61 cases of serious infection and 16 outbreaks in nurseries. Rev Infect Dis 8, 918-926 (1986).

9. S. Morosky et al., The neonatal Fc receptor is a pan-echovirus receptor. Proc Natl Acad Sci U S A 116, 3758-3763 (2019).

10. X. Zhao et al., Human Neonatal Fc Receptor Is the Cellular Uncoating Receptor for Enterovirus B. Cell 177, 1553-1565 e1516 (2019).

11. M. Pyzik, T. Rath, W. I. Lencer, K. Baker, R. S. Blumberg, FcRn: The Architect Behind the Immune and Nonimmune Functions of IgG and Albumin. J Immunol 194, 4595-4603 (2015).

12. M. Pyzik et al., Hepatic FcRn regulates albumin homeostasis and susceptibility to liver injury. Proc Natl Acad Sci U S A 114, E2862-E2871 (2017).

13. C. Chaudhury et al., The major histocompatibility complex-related Fc receptor for IgG (FcRn) binds albumin and prolongs its lifespan. J Exp Med 197, 315-322 (2003).

14. Y. Zhang, W. M. Pardridge, Mediated efflux of IgG molecules from brain to blood across the blood-brain barrier. J Neuroimmunol 114, 168-172 (2001).

15. F. Schlachetzki, C. Zhu, W. M. Pardridge, Expression of the neonatal Fc receptor (FcRn) at the blood-brain barrier. J Neurochem 81, 203-206 (2002).

16. J. Ahn et al., Susceptibility of mouse primary cortical neuronal cells to coxsackievirus $B$. J Gen Virol 85, 1555-1564 (2004).

17. R. Feuer et al., Coxsackievirus targets proliferating neuronal progenitor cells in the neonatal CNS. J Neurosci 25, 2434-2444 (2005).

18. R. Feuer et al., Coxsackievirus B3 and the neonatal CNS: the roles of stem cells, developing neurons, and apoptosis in infection, viral dissemination, and disease. Am J Pathol 163, 1379-1393 (2003).

19. S. A. Hughes, H. M. Thaker, V. R. Racaniello, Transgenic mouse model for echovirus myocarditis and paralysis. Proc Natl Acad Sci U S A 100, 15906-15911 (2003).

20. G. Zhang et al., Pathological Features of Echovirus-11-Associated Brain Damage in Mice Based on RNA-Seq Analysis. Viruses 13 (2021).

21. A. I. Wells et al., Human FcRn expression and Type I Interferon signaling control Echovirus 11 pathogenesis in mice. PLoS Pathog 17, e1009252 (2021).

22. M. Nishikawa et al., Abnormalities of brain perfusion in echovirus type 30 meningitis. $J$ Neurol Sci 179, 122-126 (2000). 
23. S. M. Wang et al., Cerebrospinal fluid cytokines in enterovirus 71 brain stem encephalitis and echovirus meningitis infections of varying severity. Clin Microbiol Infect 13, 677-682 (2007).

24. T. Ichiyama et al., Analysis of cytokine levels in cerebrospinal fluid in mumps meningitis: comparison with echovirus type 30 meningitis. Cytokine 30, 243-247 (2005).

25. T. Chonmaitree, S. Baron, Bacteria and viruses induce production of interferon in the cerebrospinal fluid of children with acute meningitis: a study of 57 cases and review. Rev Infect Dis 13, 1061-1065 (1991).

26. H. Singh, J. Koury, M. Kaul, Innate Immune Sensing of Viruses and Its Consequences for the Central Nervous System. Viruses 13 (2021).

27. H. M. Lazear et al., Interferon-lambda restricts West Nile virus neuroinvasion by tightening the blood-brain barrier. Sci Transl Med 7, 284 ra259 (2015).

28. Y. Li et al., Interferon-lambda Attenuates Rabies Virus Infection by Inducing InterferonStimulated Genes and Alleviating Neurological Inflammation. Viruses 12 (2020).

29. D. C. Roopenian, G. J. Christianson, G. Proetzel, T. J. Sproule, Human FcRn Transgenic Mice for Pharmacokinetic Evaluation of Therapeutic Antibodies. Methods Mol Biol 1438, 103-114 (2016).

30. A. I. Wells, K. A. Grimes, C. B. Coyne (2022) Enterovirus replication and dissemination are differentially controlled by type I and III interferons in the GI tract.

31. H. M. T. Choi et al., Third-generation in situ hybridization chain reaction: multiplexed, quantitative, sensitive, versatile, robust. Development 145 (2018).

32. H. M. Choi et al., Mapping a multiplexed zoo of mRNA expression. Development 143, 3632-3637 (2016).

33. P. R. Cooper et al., Efflux of monoclonal antibodies from rat brain by neonatal Fc receptor, FcRn. Brain Res 1534, 13-21 (2013).

34. S. Latvala, B. Jacobsen, M. B. Otteneder, A. Herrmann, S. Kronenberg, Distribution of FcRn Across Species and Tissues. J Histochem Cytochem 65, 321-333 (2017).

35. J. DeSisto et al., Single-Cell Transcriptomic Analyses of the Developing Meninges Reveal Meningeal Fibroblast Diversity and Function. Dev Cell 54, 43-59 e44 (2020).

36. K. Kierdorf, T. Masuda, M. J. C. Jordao, M. Prinz, Macrophages at CNS interfaces: ontogeny and function in health and disease. Nat Rev Neurosci 20, 547-562 (2019).

37. T. Matsubara et al., Mononuclear cells and cytokines in the cerebrospinal fluid of echovirus 30 meningitis patients. Scand J Infect Dis 32, 471-474 (2000).

38. C. G. Drummond et al., Enteroviruses infect human enteroids and induce antiviral signaling in a cell lineage-specific manner. Proc Natl Acad Sci U S A 114, 1672-1677 (2017).

39. M. C. Freeman et al., Respiratory and intestinal epithelial cells exhibit differential susceptibility and innate immune responses to contemporary EV-D68 isolates. Elife 10 (2021).

40. C. Good, A. I. Wells, C. B. Coyne, Type III interferon signaling restricts enterovirus 71 infection of goblet cells. Sci Adv 5, eaau4255 (2019).

41. S. K. Kuss, C. A. Etheredge, J. K. Pfeiffer, Multiple host barriers restrict poliovirus trafficking in mice. PLoS Pathog 4, e1000082 (2008).

42. B. D. Bultmann, H. J. Eggers, J. Galle, O. Haferkamp, Age dependence of paralysis induced by echovirus type 9 in infant mice. J Infect Dis 147, 999-1005 (1983).

43. N. M. O'Brown, S. J. Pfau, C. Gu, Bridging barriers: a comparative look at the bloodbrain barrier across organisms. Genes Dev 32, 466-478 (2018).

44. A. G. Wu et al., Age-dependent susceptibility to reovirus encephalitis in mice is influenced by maturation of the type-I interferon response. Pediatr Res 83, 1057-1066 (2018). 
576

577

578

579

580

581

582

583

584

585

586

587

588

589

590

591

592

593

594

595

596

597

598

599

600

601

45. D. Lyden, J. Olszewski, S. Huber, Variation in susceptibility of Balb/c mice to coxsackievirus group B type 3-induced myocarditis with age. Cell Immunol 105, 332-339 (1987).

46. R. Khatib, J. L. Chason, B. K. Silberberg, A. M. Lerner, Age-dependent pathogenicity of group B coxsackieviruses in Swiss-Webster mice: infectivity for myocardium and pancreas. J Infect Dis 141, 394-403 (1980).

47. X. Qiu, M. Z. Wang, Quantification of Neonatal Fc Receptor and Beta-2 Microglobulin in Human Liver Tissues by Ultraperformance Liquid Chromatography-Multiple Reaction Monitoring-based Targeted Quantitative Proteomics for Applications in Biotherapeutic Physiologically-based Pharmacokinetic Models. Drug Metab Dispos 48, 925-933 (2020).

48. S. Morosky, N. J. Lennemann, C. B. Coyne, BPIFB6 Regulates Secretory Pathway Trafficking and Enterovirus Replication. J Virol 90, 5098-5107 (2016).

49. M. S. Oberste, W. A. Nix, K. Maher, M. A. Pallansch, Improved molecular identification of enteroviruses by RT-PCR and amplicon sequencing. J Clin Virol 26, 375-377 (2003). 

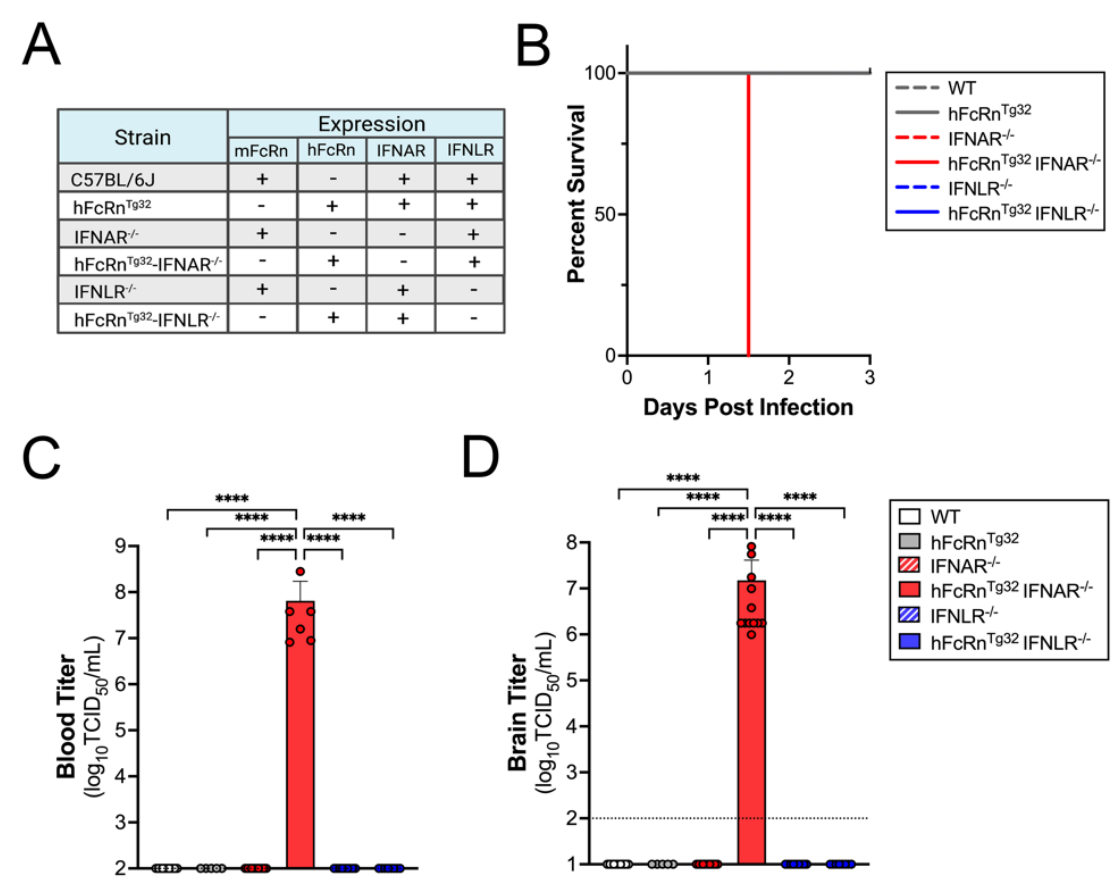

D
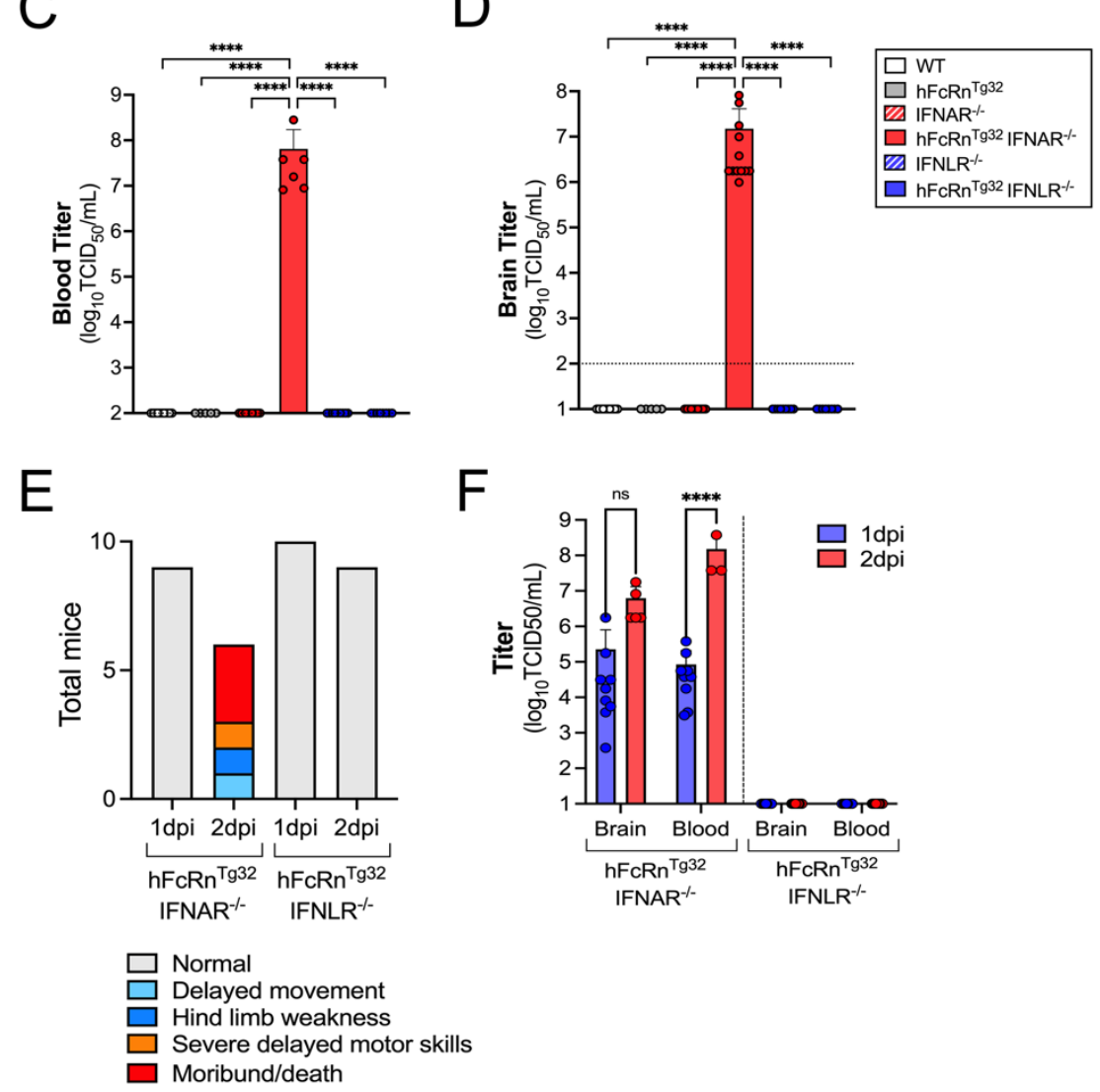

F

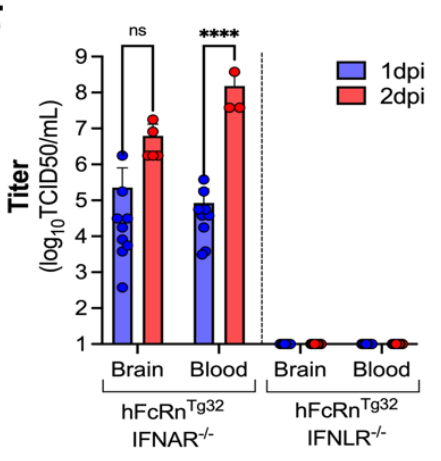

603

604

605

606

607

608

609

610

611

612

613

614

615
Figure 1. Ablation of type I interferon signaling and human FcRn expression are required for echovirus infection in the brain. (A) Table of the six genotypes used in this study. Shown is the expression of mouse or human FcRn, IFNAR, and IFNLR amongst these genotypes. (B) Survival of the indicated genotype of mice inoculated with $10^{4}$ PFU E5 by the IP route and monitored for 3 days post-inoculation. The number of pups in each genotype are as follows: WT

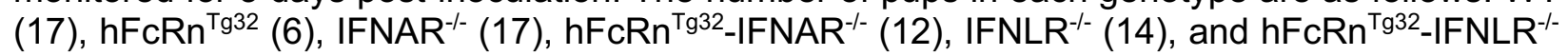
(8). The log-rank test was used to analyze the statistical difference of the survival rate. (C and D). At 3dpi, animals were sacrificed and viral titers in blood (C) and brain (D) determined by TCID50 assays. Titers are shown as $\log _{10} \mathrm{TCID} 50 / \mathrm{mL}$ with the limit of detection indicated by a dotted line. Data are shown as mean \pm standard deviation with individual animals shown as each data point. Data are shown with significance determined with a Kruskal-Wallis test with a Dunn's test for 
616 multiple comparisons. (E and F) hFcRn ${ }^{T g 32}$-IFNAR ${ }^{-/-}$and $\mathrm{hFCRn}^{\text {Tg32 }}$ IFNLR $^{-/-}$mice were inoculated 617 with $10^{3}$ PFU of E5 by the IP route and monitored for signs of disease. (E) Clinical symptoms 618 observed of $\mathrm{hFcRn}^{\mathrm{Tg} 32}$-IFNAR $^{-/-}$or $\mathrm{hFCRn}^{\mathrm{Tg} 32}$-IFNLR $^{-/-}$pups at either one- or two-days post 619 infection. (F) At 1 or $2 \mathrm{dpi}$, animals were sacrificed and viral titers in blood and brain determined by TCID50 assays. Titers are shown as $\log _{10} T C I D 50 / \mathrm{mL}$ with the limit of detection indicated by a dotted line. Data are shown as mean \pm standard deviation with individual animals shown as each data point. Data are shown with significance determined with a Two-way Anova with Šídák's multiple comparisons tests $\left({ }^{*} p<0.05,{ }^{* *} p<0.005,{ }^{* * *} p<0.0005,{ }^{* * * *} p<0.0001\right)$. 

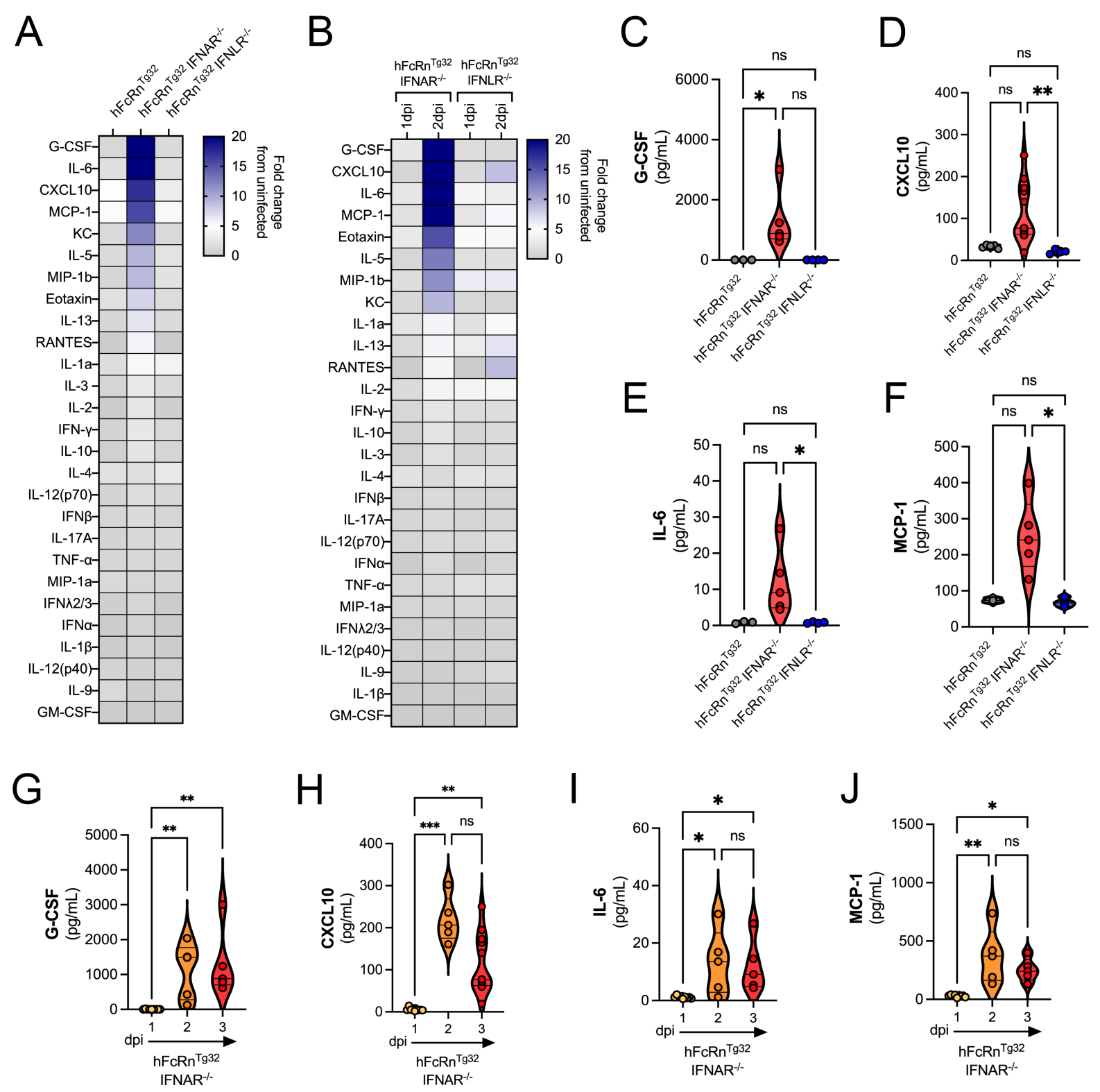

Figure 2. Immunological signature of echovirus infected brains. Pups were IP inoculated and sacrificed either 1, 2, or 3dpi. Cytokine expression in brain tissue homogenates were analyzed by multiplex Luminex assays. (A) Heatmap demonstrating the induction (shown as fold-change from uninfected control) in E5-infected mice of the indicated genotype sacrificed at 3dpi. Blue denotes significantly increased cytokines in comparison to untreated. Grey or white denote little to no changes (scale at top right). (B) Heatmap demonstrating the induction (shown as fold-change from $\mathrm{hFcRn}{ }^{T g 32}$ pups) in E5-infected mice of the indicated genotype sacrificed at 1 or $2 \mathrm{dpi}$, as indicated. Blue denotes significantly increased cytokines in comparison to untreated. Grey or white denote little to no changes (scale at top right). (C-F) The top four cytokines induced from animals sacrificed at 3dpi from (A) including G-CSF (C), CXCL10 (D), IL-6 (E), MCP-1 (F) shown as $\mathrm{pg} / \mathrm{mL}$. (G-J) The top four cytokines induced in $\mathrm{hFcRn}{ }^{\mathrm{Tg} 32}-\mathrm{IFNAR}^{-/-}$animals over the course of 
639 the infection time. Shown are G-CSF (G), CXCL10 (H), IL-6 (I), and MCP-1 (J). Data are shown 640 as mean \pm standard deviation and individual animals (points). Data are shown with significance 641 determined with a Kruskal-Wallis test with a Dunn's test for multiple comparisons $\left({ }^{*} \mathrm{p}<0.05\right.$, $642 \quad{ }^{* *} p<0.005,{ }^{* * *} p<0.0005,{ }^{* * * *} p<0.0001$, ns-not significant).

643

644

645

646

647

648

649

650 

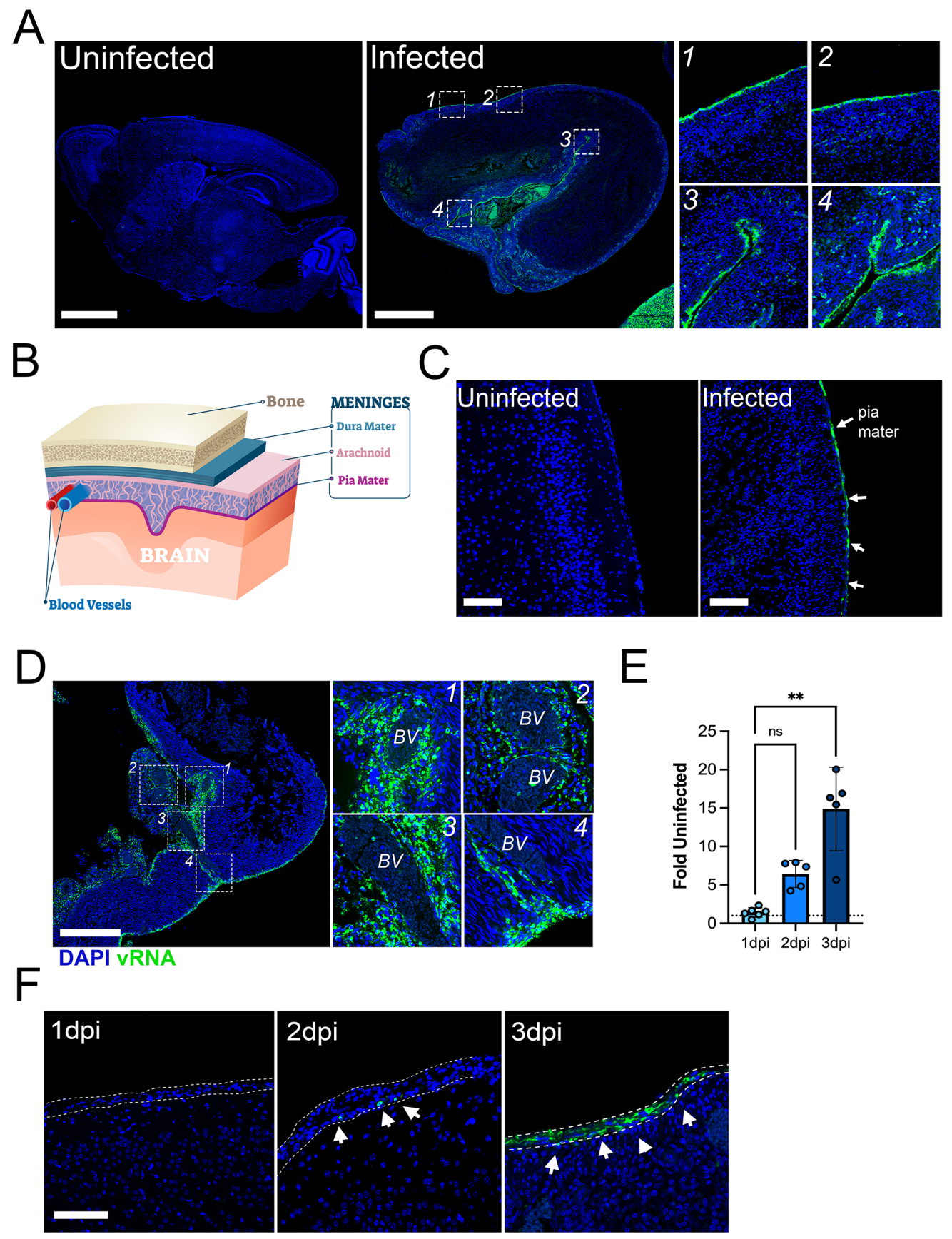

DAPI vRNA

Figure 3. Echovirus replication in the leptomeninges. (A) Tile scan of the brains from an uninfected animal (left) and $\mathrm{E} 5$ infected $\mathrm{hFCRn}{ }^{\mathrm{Tg} 32}-\mathrm{IFNAR}^{-1-}$ animal at $3 \mathrm{dpi}$ (middle) using Hybridization chain reaction RNA-FISH (HCR) for vRNA (in green) and DAPI (in blue). Numbered white boxes show zoomed areas to the right. (B) Schematic representation of the different layers of the meninges surrounding the brain. The dura mater in teal with the leptomeninges (arachnoid and pia mater) in shades of pink. (C) HCR for vRNA (in green) and DAPI (in blue) of a brain from 
660

661

662

663

664

665

666

667

668

669

670

671

672

673

674

675

676 an uninfected or an E5 infected hFcRn ${ }^{\text {Tg32-IFNAR }}{ }^{-/}$animal at 3dpi. White arrows denote areas of vRNA. (D) Tile scan of HCR for E5 vRNA from a brain of an infected hFcRn ${ }^{T g 32}{ }_{\text {-IFNAR }}{ }^{-1-}$ animal at $3 \mathrm{dpi}$ of areas of infection surrounding blood vessels (BV). DAPI-stained nuclei in blue and VRNA in green. White boxes indicate zoomed images at right with numbers in the top right corner denoting the corresponding zoomed image. (E) Image analysis of the extent of vRNA signal in the brains of hFcRn ${ }^{\text {Tg32 }}$-IFNAR ${ }^{-/}$animals at $1-3 \mathrm{dpi}$ as shown as a fold change from uninfected controls. Symbols represent unique regions used in quantification. Data are shown with significance determined with a Kruskal-Wallis test with a Dunn's test for multiple comparisons ( ${ }^{* *} p<0.01$, ns-not significant). (F) HCR for vRNA (in green) and DAPI (in blue) of the brains from infected $\mathrm{hFcRn}{ }^{\mathrm{Tg} 32}$-IFNAR ${ }^{-/-}$animals at 1, 2, or 3dpi, as indicated at top left. Dotted lines highlight the leptomeninges and arrows denote infected cells. Scale bars are as follows: $1 \mathrm{~mm}(\mathrm{~A}), 100 \mu \mathrm{m}$ (C), $1 \mathrm{~mm}(\mathrm{D})$, and $50 \mu \mathrm{m}(\mathrm{F})$. 


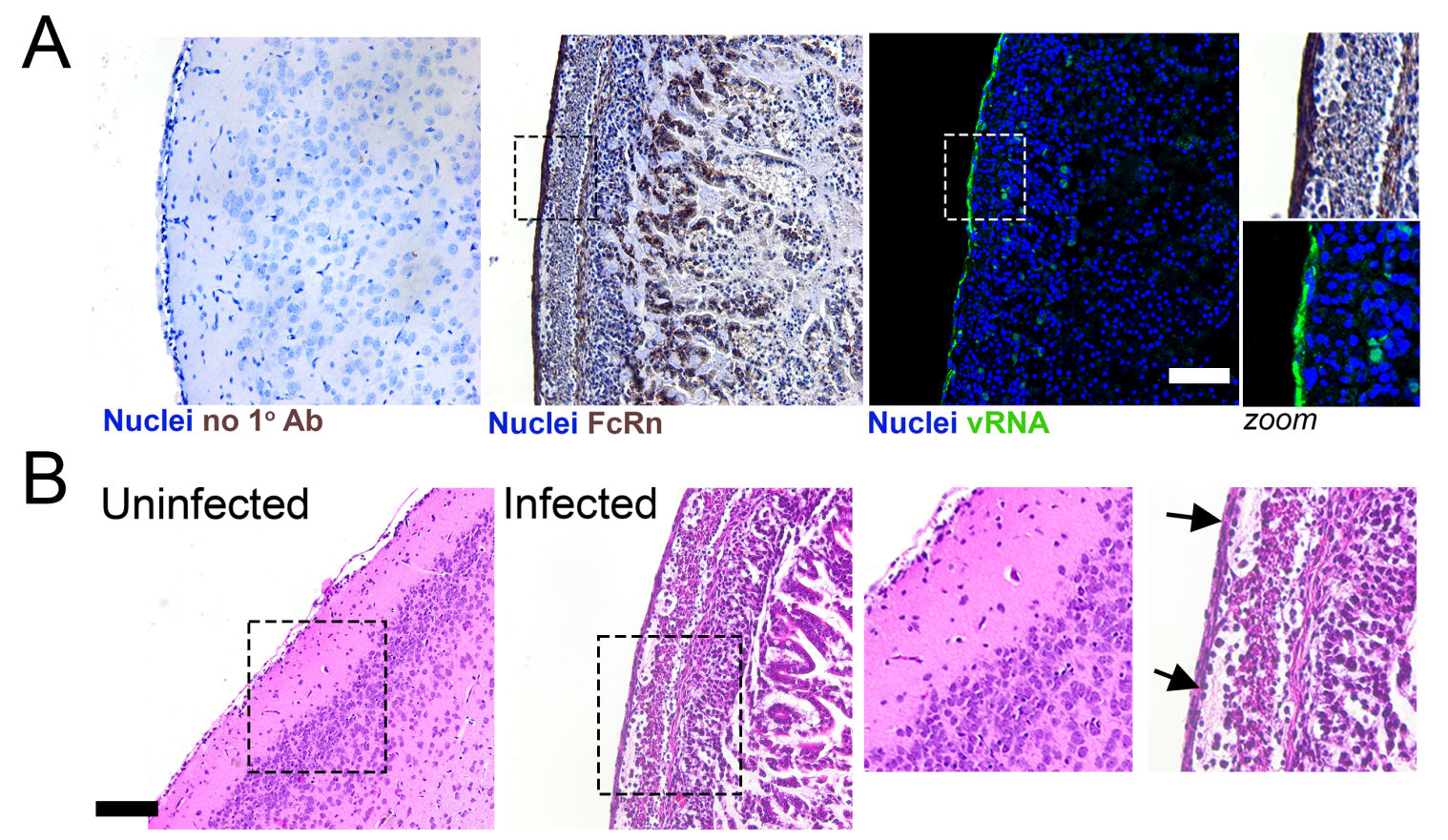

\section{C}
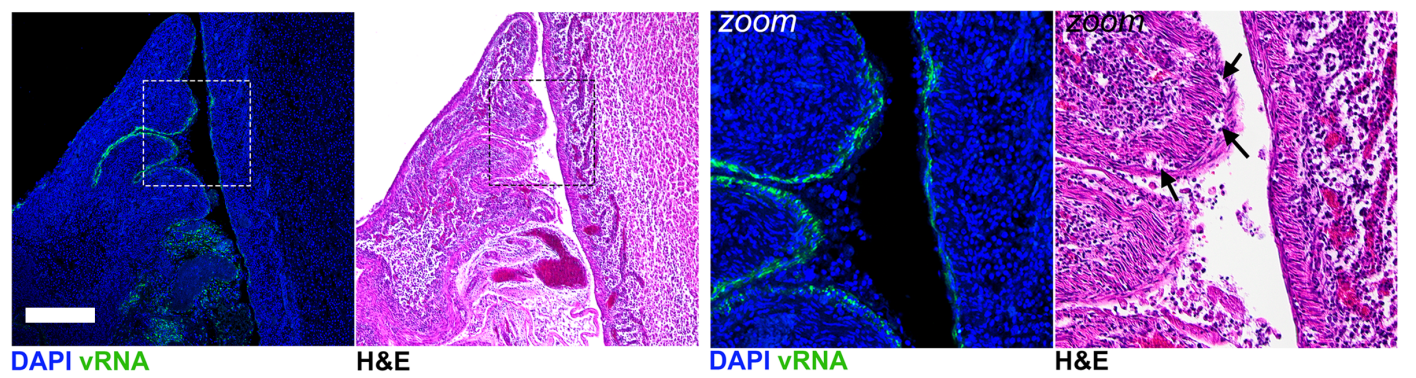

D
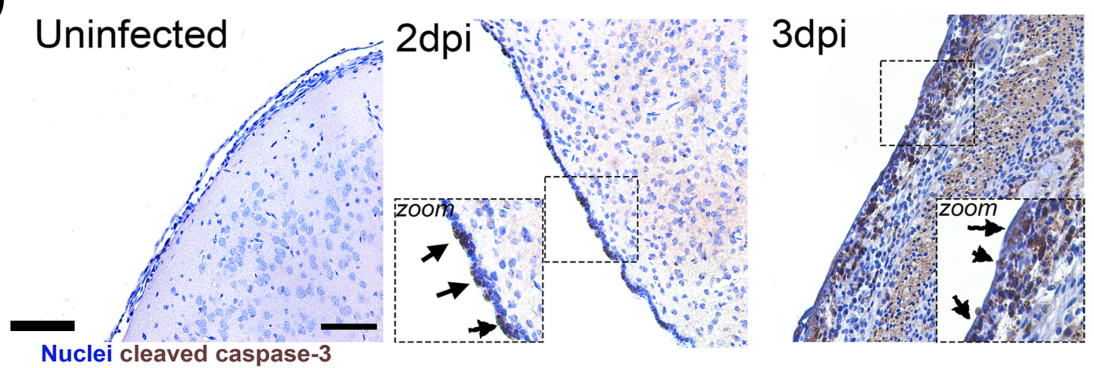

Figure 4. Echovirus replication in the meninges induces inflammation and cell death. (A), Immunohistochemistry for $\mathrm{hFcRn}$ and $\mathrm{HCR}$ for $\mathrm{VRNA}$ in the brain of a $\mathrm{hFCRn} \mathrm{n}^{\mathrm{Tg} 32}$-IFNAR ${ }^{-/-}$animal infected with E5 for 3d. At left, no primary antibody control in an uninfected control animal. Middle, $\mathrm{hFcRn} \mathrm{IHC}$ and right, HCR for vRNA (in green) and DAPI (in blue) from the same E5 infected $\mathrm{hFcRn}{ }^{\mathrm{Tg} 32}$-IFNAR ${ }^{-1-}$ animal. Boxes indicate regions that are zoomed at right. (B) Hematoxylin and eosin staining of representative brain sections from an uninfected or E5 infected $\mathrm{hFCRn}^{{ }^{\mathrm{Tg}} 32}$ $\mathrm{IFNAR}^{-1 /}$ animal. Black boxes show areas of zoomed images at right. Arrows denote regions of inflammation within the meninges. (C) HCR for E5 VRNA or hematoxylin and eosin staining of the cerebellum within the brain of a $\mathrm{hFCRn}^{\text {Tg32 }}$ IFNAR $^{-1-}$ animal at $3 \mathrm{dpi}$. HCR shows VRNA (in green) and DAPI (in blue). Boxes denote areas of zoomed images at right. Arrows denote region of 
689 inflammation within the meninges. (D) IHC for cleaved caspase 3 in uninfected, or hFcRn ${ }^{T g 32}$ 690 IFNAR $^{-/}$animals infected with E5 for $2 \mathrm{dpi}$, or $3 \mathrm{dpi}$. Arrows denote cells that are positive for 691 cleaved caspase 3. Black boxes show zoomed images. Scale bars are as follows: $100 \mu \mathrm{m}(\mathrm{A}, \mathrm{B}$, 692 D) and $1 \mathrm{~mm}(\mathrm{C})$.

693

694

695

696 

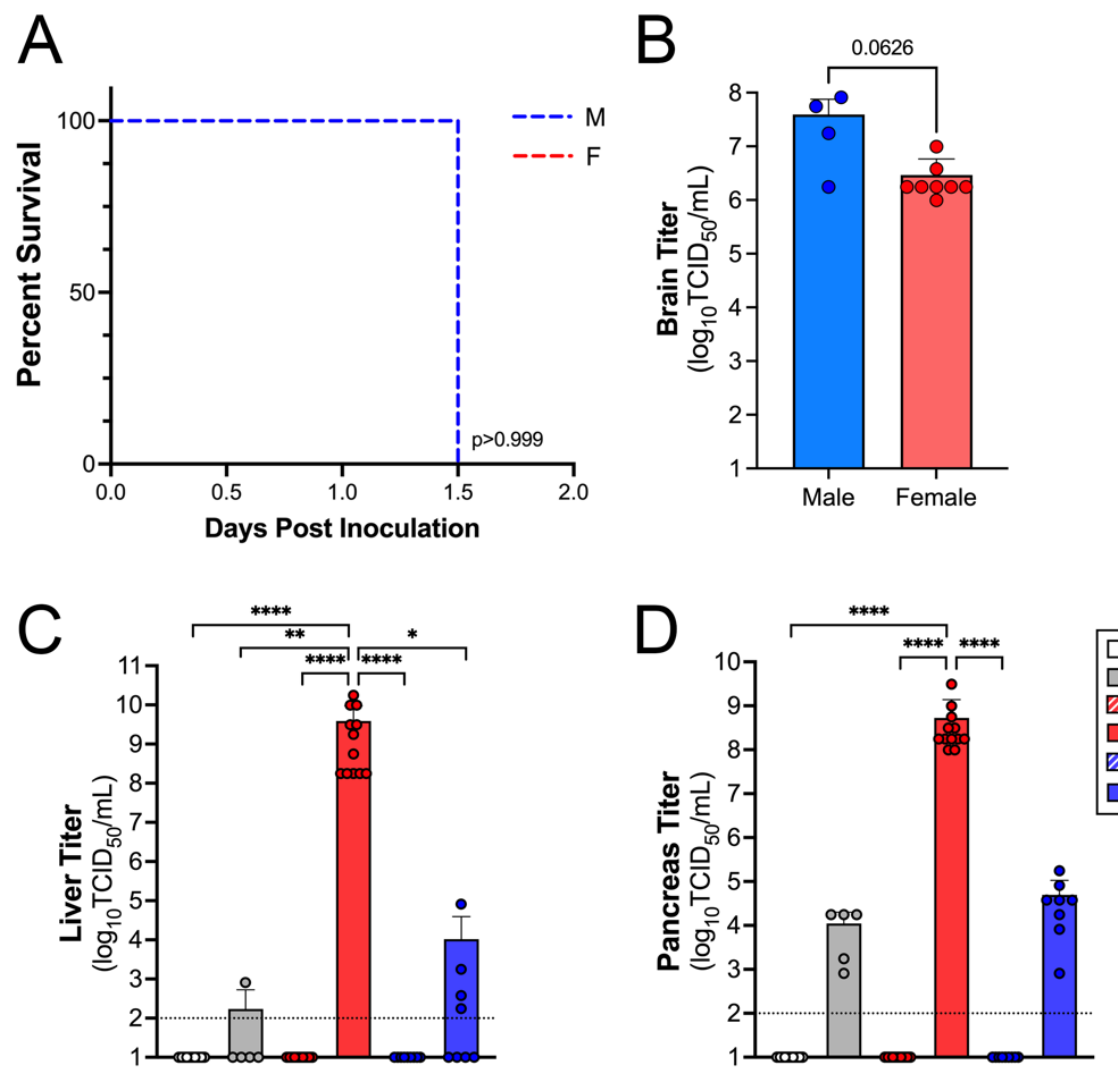

Supplemental Figure 1. (A) Survival of $h F c R n^{T g 32}$-IFNAR ${ }^{-/-}$mice inoculated with $10^{4}$ PFU of E5 by the IP route and monitored for 3 days post-inoculation. Animals are broken down by sex (Mmale, F-female) to represent any potential sex differences in mortality. The log-rank test was used to analyze the statistical difference of the survival rate with $p$ value shown. (B) Brain titers from

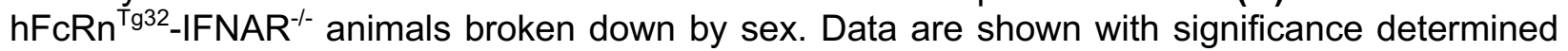
with a Mann-Whitney $U$ test with $p$ value shown. (C and D) The indicated genotype of mice inoculated with $10^{4}$ E5 by the IP route. At $3 \mathrm{dpi}$, animals were sacrificed and viral titers in liver (C) and pancreas (D) determined by TCID50 assays. Titers are shown as $\log _{10}$ TCID50/mL with the limit of detection indicated by a dotted line. Data are shown as mean \pm standard deviation with individual animals shown as each data point. Data are shown with significance determined with a Kruskal-Wallis test with a Dunn's test for multiple comparisons $\left({ }^{*} p<0.05,{ }^{* *} p<0.005,{ }^{* *} p<0.0005\right.$, $\left.{ }^{* * *} p<0.0001\right)$. 


\section{Supplemental Figure 2}

A

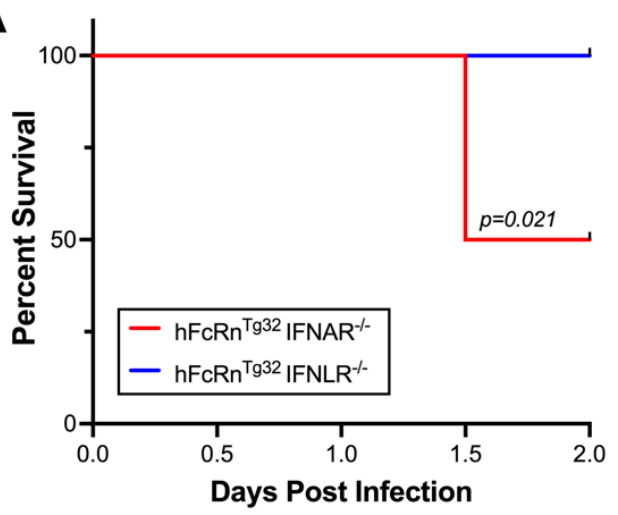

C

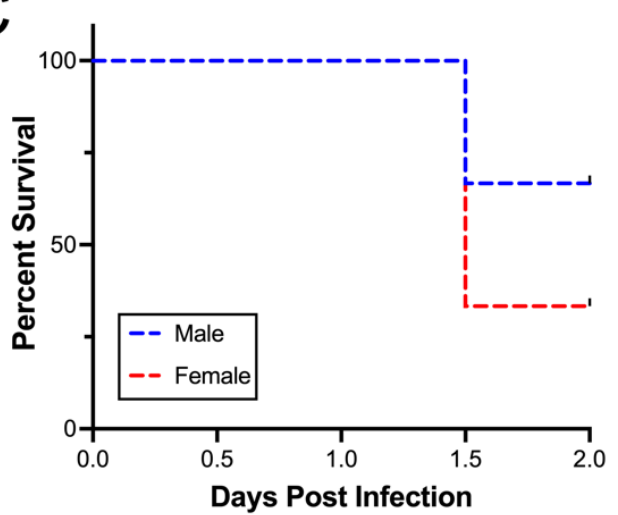

B

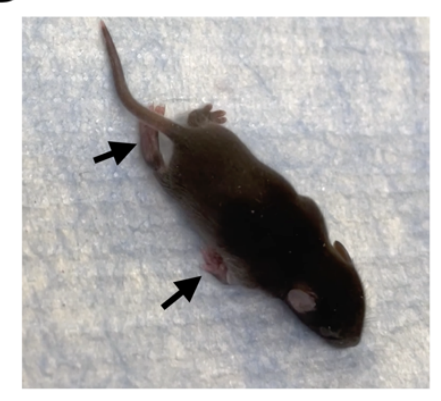

D

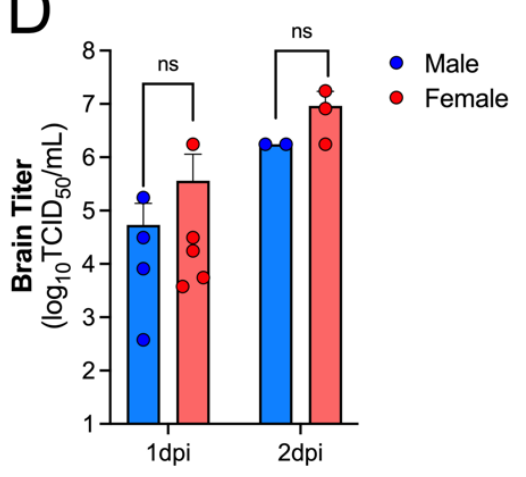

Supplemental Figure 2. (A) Survival of $h F c R n^{T 932}-I F N A R^{-1-}$ and $h F c R n^{T g 32}-I F N L R^{-1-}$ mice which were inoculated with $10^{3} \mathrm{PFU}$ of E5 by the IP route and monitored for 2 days post-inoculation. The number of pups in each genotype are as follows: $\mathrm{hFcRn}^{\text {Tg32 }}$ IFNAR $^{-1-}$ (6) and $\mathrm{hFcRn}{ }^{\text {Tg32 }}$ IFNLR ${ }^{-1-}(9)$. The log-rank test was used to analyze the statistical difference of the survival rate of

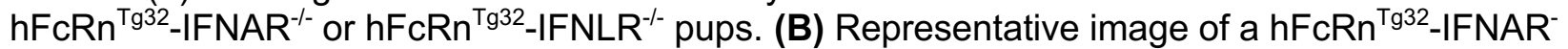
1- mouse with hemiplegia. Arrows denote limbs that are impacted. (C) Survival of $\mathrm{hFcRn}^{\mathrm{Tg}^{32}}$. IFNAR $^{-/}$mice broken down by sex which were inoculated with $10^{3}$ PFU of E5 by the IP route and monitored for 2 days post-inoculation. The log-rank test was used to analyze the statistical difference of the survival rate of between males and females with the $p$ value shown. (D) Brain titers from $\mathrm{hFcRn}^{\mathrm{Tg} 32}$-IFNAR ${ }^{-/-}$animals broken down by sex at either 1 or $2 \mathrm{dpi}$. Data are shown with significance determined with a Mann-Whitney $\mathrm{U}$ test, ns-not significant. 


\section{Supplemental Figure 3}
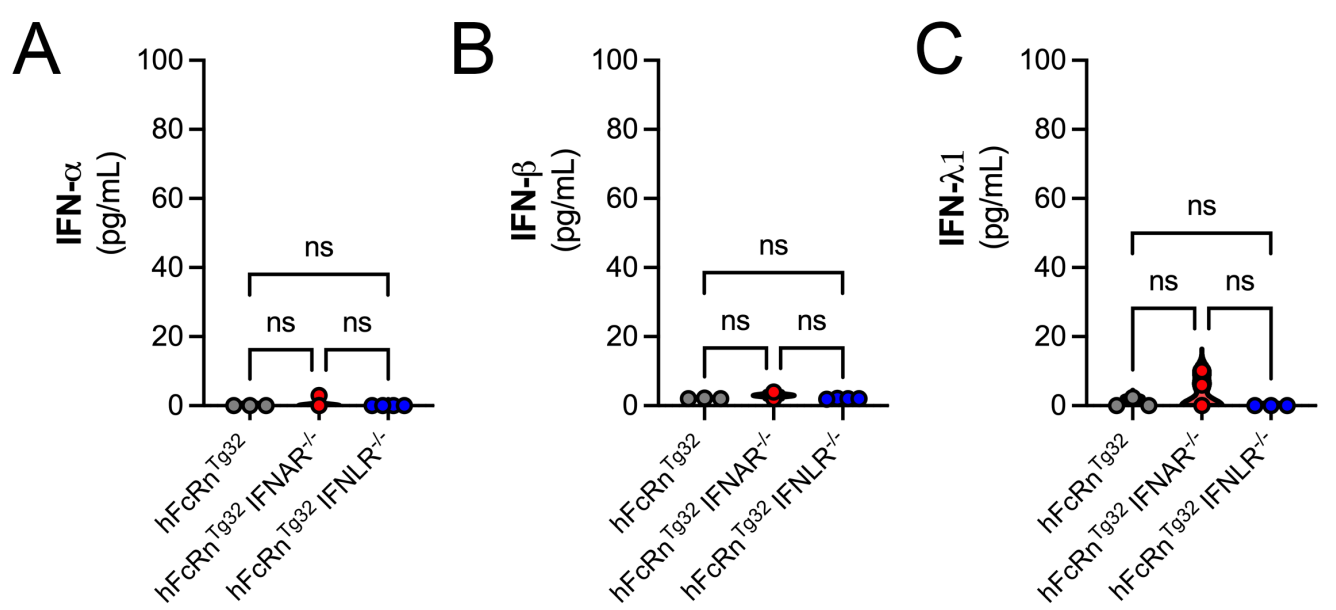

$D$
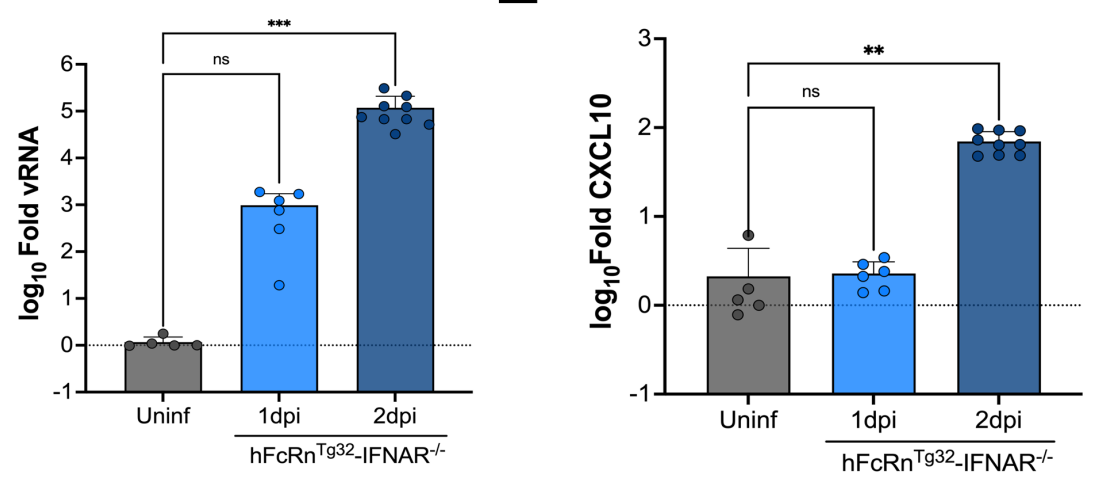

Supplemental Figure 3. (A-C) Luminex multiplex assays from brain tissue homogenates of

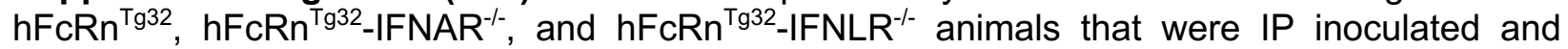
sacrificed at 3dpi. Shown as the concentration in $\mathrm{pg} / \mathrm{mL}$ IFN- $\alpha$ (A), IFN- $\beta$ (B), and IFN 1 1 (C). RTqPCR for viral RNA (D) or CXCL10 (E) from the brains of uninfected, 1dpi, or $2 \mathrm{dpi}_{\mathrm{hFCRn}}{ }^{\mathrm{Tg} 32}$ IFNAR $^{-/}$animals. Data are shown with the significance determined with a Kruskal-Wallis test with a Dunn's test for multiple comparisons $\left({ }^{* *} p<0.005,{ }^{* * *} p<0.0005\right.$, ns-not significant). 


\section{Supplemental Figure 4}
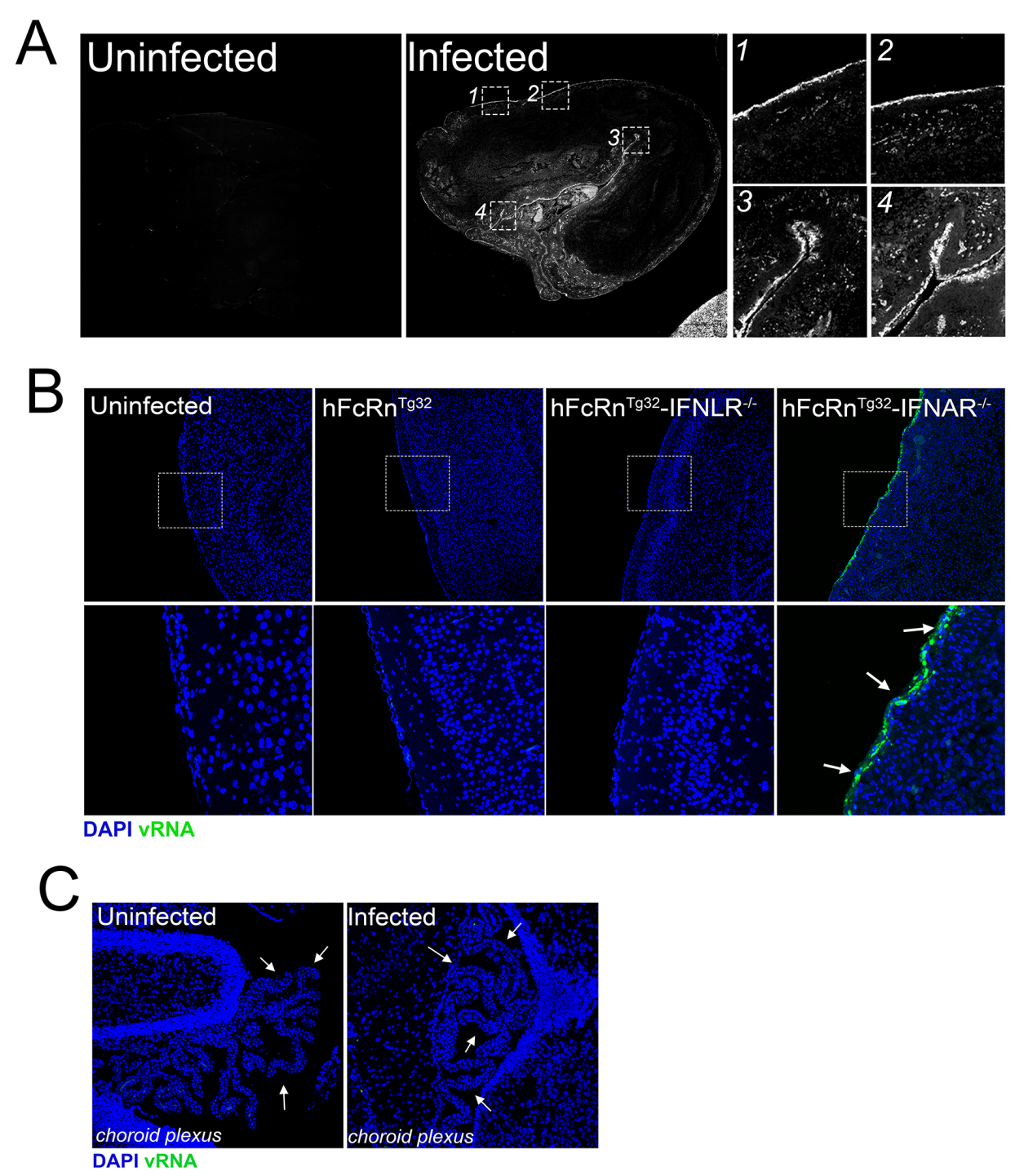

Supplemental Figure 4. (A) Tile scan of the brains from an uninfected (left) and E5 inoculated at $3 \mathrm{dpi}$ (middle) $\mathrm{hFcRn}^{\mathrm{Tg} 32}$-IFNAR $^{-{ }_{-}}$animal using Hybridization chain reaction RNA-FISH (HCR) from figure $4 A$. vRNA is shown in white. (B) HCR of brain sections from $\mathrm{hFcRn}^{\mathrm{Tg} 32}, \mathrm{hFcRn}^{\mathrm{Tg} 32}$ IFNAR $^{-/-}$, and $\mathrm{hFCRn}^{\text {Tg32 }}$ IFNLR $^{-/-}$animals at 3dpi. E5 viral RNA (VRNA) is shown in green and DAPI-stained nuclei are shown in blue. White box is shown as a zoomed image below. White arrows at right denote areas of E5 vRNA. (C) Choroid plexus region within the brain from an uninfected (left) and E5 inoculated at 3dpi (right) hFcRn ${ }^{T g 32}$-IFNAR ${ }^{-/-}$animal using HCR with vRNA (in green) and DAPI (in blue). White arrows show the choroid plexus. 
779 Supplemental Table 1. RT-qPCR Primers used in this study

780

\begin{tabular}{|l|l|l|}
\hline \multicolumn{1}{|c|}{ Target } & \multicolumn{1}{c|}{ Forward Primer } & \multicolumn{1}{c|}{ Reverse Primer } \\
\hline GAPDH & AGGTCGGTGTGAACGGATTTG & TGTAGACCATGTAGTTGAGGTCA \\
\hline Echovirus 5 & ACCCTACYGYAYTAACCGAAC & CCGCACCGAAYGCGGAKAATTTAC \\
\hline CXCL10 & AAAAAGGTCTAAAAGGGCTC & AATTAGGACTAGCCATCCAC \\
\hline
\end{tabular}

781

782 
Supplemental Table 2. Echovirus 5 HCR Probes

\begin{tabular}{|l|l|l|}
\hline $\begin{array}{l}\text { Probe } \\
\text { Pair }\end{array}$ & & \\
\hline B4P1 & $\begin{array}{l}\text { CCTCAACCTACCTCCAACAAGG } \\
\text { GCTCAGTAAACTTTCCCGGGTCT }\end{array}$ & $\begin{array}{l}\text { CATGGATTTGATCATAAGGTCCTT } \\
\text { CATTCTCACCATATTCGCTTC }\end{array}$ \\
\hline B4P2 & $\begin{array}{l}\text { CCTCAACCTACCTCCAACAAAGC } \\
\text { CCTGATGGAACTTAGATGCGTT }\end{array}$ & $\begin{array}{l}\text { CTTCAGGCACACACACCACCAGG } \\
\text { AGATTCTCACCATATTCGCTTC }\end{array}$ \\
\hline B4P3 & $\begin{array}{l}\text { CCTCAACCTACCTCCAACAAGCA } \\
\text { TTACTATGGTGGCACAATTGTT }\end{array}$ & $\begin{array}{l}\text { TGTCCATCGGTACGCTATTAATGT } \\
\text { AATTCTCACCATATTCGCTTC }\end{array}$ \\
\hline B4P4 & $\begin{array}{l}\text { CCTCAACCTACCTCCAACAATGC } \\
\text { TGCACCATCCTATAGTGGGTTT }\end{array}$ & $\begin{array}{l}\text { ATGTAGCCTGCTGCTGAGTATTCA } \\
\text { TATTCTCACCATATTCGCTTC }\end{array}$ \\
\hline B4P5 & $\begin{array}{l}\text { CCTCAACCTACCTCCAACAAGTT } \\
\text { ATGCTCAGACTTGGGCACGTCA }\end{array}$ & $\begin{array}{l}\text { TTGGCCAAAGGCTCCATATGTGTG } \\
\text { CATCTCACCATATTCGCTTC }\end{array}$ \\
\hline B4P6 & $\begin{array}{l}\text { CCTCAACCTACCTCCAACAAGTG } \\
\text { ATCAGGTCATCGTGATTCTTA }\end{array}$ & $\begin{array}{l}\text { CCAATGAGGGCTAGTGTGGCAGT } \\
\text { CAATTCTCACCATATTCGCTTC }\end{array}$ \\
\hline B4P7 & $\begin{array}{l}\text { CCTCAACCTACCTCCAACAAGAT } \\
\text { CTTCCAATTAGATTTGTTGCTA }\end{array}$ & $\begin{array}{l}\text { ACTGAGCTGTTAAGCTTTTCAGCT } \\
\text { AATTCTCACCATATTCGCTTC }\end{array}$ \\
\hline B4P8 & $\begin{array}{l}\text { CCTCAACCTACCTCCAACAACTC } \\
\text { CCTGTATAACGGTGGTCCCTGA }\end{array}$ & $\begin{array}{l}\text { TGTCTCTGGTGCAACACTAATTTT } \\
\text { GATTCTCACCATATTCGCTTC }\end{array}$ \\
\hline B4P9 & $\begin{array}{l}\text { CCTCAACCTACCTCCAACAATTA } \\
\text { GTGGGCGTGCCGCCCAGGTTTA }\end{array}$ & $\begin{array}{l}\text { GTGGGGAAGTTATACATGAGCATT } \\
\text { CATTCTCACCATATTCGCTTC }\end{array}$ \\
\hline B4P10 & $\begin{array}{l}\text { CCTCAACCTACCTCCAACAAAGC } \\
\text { GCTTCAAGACCCTCAGTACCGT }\end{array}$ & $\begin{array}{l}\text { TGGGTAACCGGCGCTCGTTGTTA } \\
\text { GATATTCTCACCATATTCGCTTC }\end{array}$ \\
\hline B4P11 & $\begin{array}{l}\text { CCTCAACCTACCTCCAACAATGC } \\
\text { ACAAGTAGTCAATGTAGTTAGT }\end{array}$ & $\begin{array}{l}\text { GCTTGTCTCTGTACAGGTGATGG } \\
\text { GAATTCTCACCATATTCGCTTC }\end{array}$ \\
\hline B4P12 & $\begin{array}{l}\text { CCTCAACCTACCTCCAACAATTT } \\
\text { CACGAATGTTGATCTCCCACCT }\end{array}$ & $\begin{array}{l}\text { TCTCACACTTTCTACGTAGTTGCA } \\
\text { CATTCTCACCATATTCGCTTC }\end{array}$ \\
\hline
\end{tabular}

